\title{
Nanostructure Characterization of Conical Silicon Carbide Nanowires and Heterostructures Induced by Release Catalysis
}

\author{
Zhenyu Liu ${ }^{*}$, V. Srot, ${ }^{* *}$ N. Y. Jin-Phillipp, ${ }^{* *}$ Peter A. van Aken, ${ }^{* *}$ M. Rühle ${ }^{* *}$ and Judith C. Yang \\ * Department of Mechanical Engineering and Materials Science Department, University of Pittsburgh, \\ Pittsburgh, PA 15261 \\ ** Max-Planck-Institute for Metals Resarch, Heisenbergstrasse 3, D-70569 Stuttgart, Germany
}

Control of nanoscale building block topology and the formation of selected polymorphs are essential for the novel application developments since their properties intimately depend on the chemical composition, size, structure, and morphology [1-6]. Nanowires represent an important and broad class of onedimensional (1D) nanostructures at the forefront of nanoscience and nanotechnology. Understanding how to control the morphology, rational design and predictable synthesis of nanowires is vital in order to deterministically integrate such nanostructures into various technologies. Shape engineering of properties represents important design flexibility for 1D nanostructures and their practical applications. Conical nanostructures have emerged as a new kind of 1D nanostructure which may be superior to nanotube, nanowire, and nanobelt structures in some aspects [7], their potential uses in field emission, in nanoscaled manipulation, and as scanning probe microscopy and near-field scanning optical microscopy probes. Tapered nanostructures are expected to have higher bending stiffness than nanotubes and nanowires and better resistance to thermal-induced drift than nanowires. Nanocones provide considerably more mechanical and thermal stability than their cylindrical counterparts because of their large bases while still affording the precision associated with small tip sizes and high aspect ratio. Recent studies revealed that the conventional assumed picture the catalyst droplet does not change during the nanowire growth is false and the catalyst diffusion during the nanowire growth can determine the length, shape and even the sidewall properties of the nanowires. The shrinkage of the metal catalyst during the nanowire evolution in high vacuum conditions leads to the consequence of Si nanocone formation [4,5].

By means of the reaction between core-shell structured carbon-encapsulated iron nanoparticles, acted as released iron catalyst, and silicon monoxide vapor generated from the mixture of silicon and silica at $1300^{\circ} \mathrm{C}$, conical $\mathrm{SiC}$ nanowires and heterostructures have been synthesized. The most important feature of our observations is the continuously changing cross-sectional diameters of the conical SiC nanowires. This is related to the enlargement of the metal catalyst due to the thermally-induced release of the iron nanoparticles from the graphite-like carbon shell and the agglomeration of the released Fe nanoclusters. The metallic core nanocrystal releases or migrates out from the graphite-like carbon shell and functions as the catalyst for the conical $\mathrm{SiC}$ nanowire formation. The catalytic droplet size during the nanowire formation process dominates the shape of the forming nanowire. The nucleated catalyst droplet for $\mathrm{SiC}$ nanowire continuously enlarges by the capture of later released iron nanocrystals to form larger catalyst seed, and then the formed nanowires become cone-shaped. We hope that this work will generate further interest in the released catalysis method and enable further developments in the shape and structural dependent properties of semiconductor conical nanowires and their heterostructure. We propose that it is an effective tactic to control the nanocatalyst size from core-shell structured nanoparticles, meanwhile, the release effect would prolong the catalytic effects of the core-shell structured nanocatalysts and eventually lead to unusual catalytic reactions.

\section{References}

[1] Zhang, G. Y.; Jiang, X.; Wang, E. G. Science 2003, 300, 472.

[2] Hanon, J. B.; Kodambaka, S.; Ross, F. M.; Tromp, R. M. Nature 2006, 440, 69.

[3] Cao, L.; Laim, L.; Ni, C.; Nabel, B.; Spanier, J. E. J. Am. Chem. Soc. 2005, 127; 13782.

[4] Cao, L.; Garipcan, B.; Atchison, J. S.; Ni, C.; Nabet, B.; Spanier, J. E. Nano Lett. 2006, 6, 1852. 
[5] Liu, C.; Hu, Z.; Wu, Q.; Wang, X.; Chen, Y.; Sang, H.; Zhu, J.; Deng, S.; Xu, N. J. Am. Chem. Soc. 2005; $127,1318$.

[6] Li, Y.; Qian, L. H. Li, W. F.; Yang, C. N.; Ma, X. L. App. Phys. Lett. 2005, 87, 183107.

[7] Liu, Z.; Ci, L.; Srot, V.; Jin-Phillipp, N.; Aken, von P.; Rühle M.; Yang, J. App. Phys. Lett. 2008, 98, 233113.

[8] This research was supported in part by grants from the NSF (Grants No. NER-0508238 and DMR0804892).


FIG. 1. (a) SEM and (b) TEM images of the initial carbon-encapsulated iron nanoparticles, showing core-shell structure. The upper inset is the select area electron diffraction (SAED) pattern of the nanoparticles. The lower inset is a HRTEM image of the iron core revealing $\alpha$-Fe structure. (c). SEM image of the resultant conical SiC nanowires. (d) A HRTEM image of the tip of the conical SiC nanowire showing a crystalline $\mathrm{SiC}$ with nanoscale twins surrounded by a thin amorphous layer. The inset shows the corresponding fast Fourier transformation of the HRTEM image revealing the nanotwin feature.
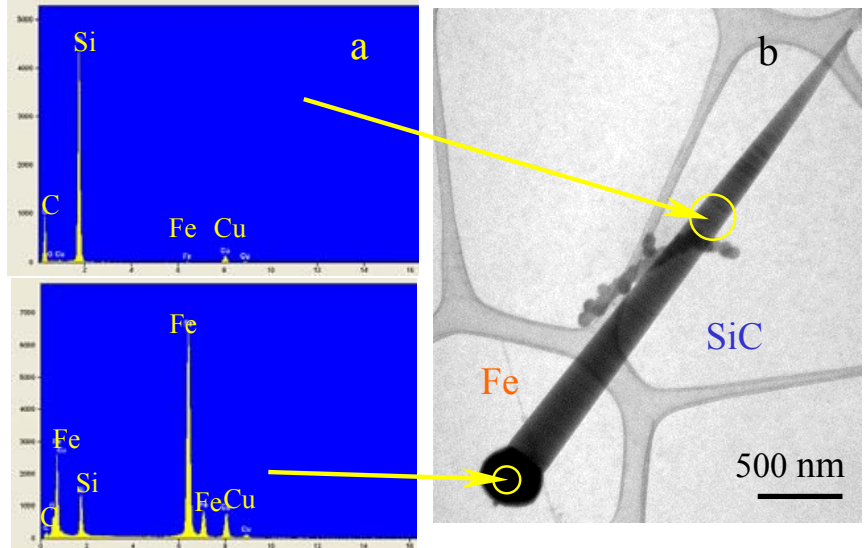

kev

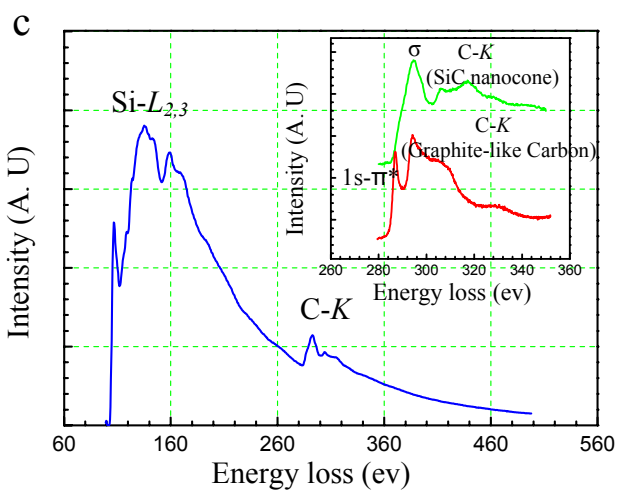

FIG. 2 EDS spectra(a) BF image of a typical conical SiC nanowire (b), and EELS spectra (c) of the conical $\mathrm{SiC}$ nanowires. 